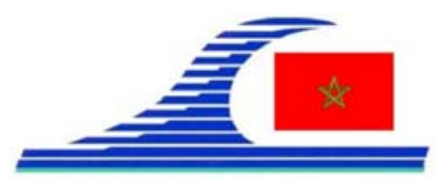

Conférence Méditerranéenne Côtière et Maritime

EDITION 2, TANGER, MAROC (2011)

Coastal and Maritime Mediterranean Conference

Disponible en ligne - http://www.paralia.fr - Available online

\title{
Evaluation de l'impact potentiel d'un gradient de salinité sur le milieu marin récepteur
}

\author{
Jean-Luc BONNEFONT ${ }^{1}$, Yvan MARTIN ${ }^{1}$, Stellio CASAS $^{2}$
}

1. Institut Océanographique Paul Ricard, Ile des Embiez, 83140 Six-Fours-les-Plages, France.labembiez@wanadoo.fr

2. Veolia Environnement Recherche et Innovation, Département "Environnement et

Santé", 10 rue Jacques Daguerre 92500 Rueil-Malmaison, France

\section{Résumé :}

Afin d'évaluer l'impact du rejet de saumures issues du dessalement de l'eau de mer sur le milieu marin, 3 séries d'expérimentations ont été conduites en mésocosmes entre juin 2009 et juin 2010. Les biotopes reconstitués dans les mésocosmes sont typiques des petits fonds côtiers méditerranéens. Il apparaît que peu d'espèces sont sensibles à l'augmentation de salinité (43 à 50), à l'exception de cnidaires ou d'annélides. Les indicateurs les plus pertinents semblent appartenir à la méiofaune benthique, avec une prédominance de nématodes dans les bassins où la salinité est la plus élevée.

\section{Mots-clés :}

Dessalement de l'eau de mer - Saumures - Mésocosmes - Macrobenthos - Méiofaune

\section{Introduction}

Les rejets de saumures issues d'usines de dessalement de l'eau de mer peuvent entraîner des modifications locales de la salinité au point de rejet et la création d'un gradient de salinité dont l'amplitude et l'étendue dépendent des conditions topographiques et hydrodynamiques du site. De tels rejets peuvent entraîner des conséquences non seulement physiques (modification de la densité de l'eau notamment au point source, création éventuelle de lentilles de saumures, modification de la solubilité de l'O2, etc.) mais aussi biologiques compte tenu des limites de tolérance des espèces du site (modification des peuplements benthiques animaux et végétaux selon leur degré d'euryhalinité, sélection/adaptation ou mortalité des composants, effets sur le plancton et les bactéries, déplacement de la faune vagile et de l'ichtyofaune selon leurs préférences osmotiques, etc.).

Dans le cadre des études d'impact à réaliser en application du Code de l'Environnement, il est apparu nécessaire d'évaluer l'ampleur de ces évènements. L'objectif de ce travail a donc été d'apporter des éléments de réponse à ces questions et développer une expertise quant à l'évaluation de l'impact potentiel d'un gradient de salinité sur le milieu marin récepteur : devenir des biocénoses dans un milieu marin modérément sur-salé (état zéro, suivi environnemental portant sur les communautés planctoniques et benthiques). 
La connaissance de la Mer :

un vecteur du développement durable en Méditerranée

Du fait de l'absence d'installation produisant des saumures en Méditerranée française, il a été convenu d'effectuer cette étude en utilisant des mésocosmes.

Trois expérimentations ont été réalisées selon ce protocole : essai $n^{\circ} 1$, juin-juillet 2009 ; essai $n^{\circ} 2$, octobre-novembre 2009 ; essai ${ }^{\circ} 3$, mai-juin 2010

\section{Description du système expérimental}

Les bassins alimentés en flux continu en eau de mer (issue du pompage servant à alimenter l'aquarium de l'Institut) et par une saumure reconstituée, permettent de reproduire un gradient de salinité dans le champ proche et le champ moyen d'un rejet par rapport à un état de référence (champ lointain).

Le choix du gradient de salinité $(48,43,40)$ a été déterminé par rapport à ce qui est généralement rencontré dans la réalité d'un rejet.

La nature des substrats implantés dans les bassins correspond également à ce qui est susceptible d'exister dans la réalité. Un rejet se fait généralement dans une zone sableuse ou sablo-vaseuse (substrat meuble) en dehors d'habitats "sensibles" (herbiers, coralligène, etc.). En s'éloignant du point de rejet, l'effluent pourra atteindre d'autres types de substrats (substrat dur).

Dans le premier bassin ( $\mathrm{E} 1, \mathrm{~S}=48)$, il a été installé un sédiment de type substrat meuble représentatif de la zone immédiatement impactée par le rejet. Dans les deux bassins suivants (E2, $S=43$ et $E 3, S=38$ ), des sédiments de type substrat meuble (comme précédemment) et substrat dur ont été reconstitués.

Deux bassins témoins (T1, T2), identiques aux précédents (mêmes temps de séjour, mêmes types de substrats, mais avec une simple circulation d'eau de mer), sont également suivis.

\section{Le biotope reconstitué dans les bassins.}

Le macrobenthos est typique des petits fonds côtiers méditerranéens jusqu'à -15 m, comportant à la fois des aires de sédiments meubles (sables) colonisés par une endofaune (ici représentée par le méiobenthos) et des substrats durs formant en Méditerranée l'habitat typique des petits fonds rocheux à algues photophiles.

Le réseau trophique ainsi mis en place comporte :

- Des producteurs (7 espèces).

- Algues vertes (chlorophycées : acétabulaires, codium, halimeda, entéromorphes).

- Algues brunes (phéophycées : padines, fucus).

- Une phanérogame (cymodocée).

- Des consommateurs primaires brouteurs (patelles, gibbules, oursins) et filtreurs (éponges, annélides, mollusques bivalves, tunicier) pour un total de 10 espèces.

- Des consommateurs secondaires prédateurs (cnidaires, étoiles de mer) et détritivores (holothuries) avec 6 espèces. 
Du fait de leur capacité de mobilité importante, les espèces vagiles (ichtyofaune, crustacés, etc.) ont été volontairement exclues de ce réseau trophique reconstitué.

\section{Résultats et discussion}

Le but de ces essais était de déterminer l'effet de fortes salinités (de 43 à 50) sur des organismes caractéristiques de la bordure littorale méditerranéenne et de tenter de définir des indicateurs pertinents d'une augmentation de la salinité.

En termes d'effet il apparaît que peu d'espèces sont sensibles à l'augmentation de salinité à l'exception de cnidaires (anémones) et d’annélides (spirographes) (tableau 1).

Tableau 1. Sensibilité des espèces représentées dans les bassins en fin d'essai (+ : présence; - : absence ; Non : espèce non présente en début d'essai) avec $P_{I}$ : producteur primaire ; C: carnivore ; $D$ : détritivore ; $F$ : filtreur ; $H$ : herbivore.

\begin{tabular}{|c|c|c|c|c|c|c|}
\hline Espèces & Nutrition & T1 & $T 2$ & E1 & $E 2$ & E3 \\
\hline Acetabularia acetabulum & $P_{I}$ & + & + & + & + & + \\
\hline Fucus virsoïdes & $P_{I}$ & + & + & + & + & + \\
\hline Enteromorpha sp. & $P_{I}$ & + & + & + & + & + \\
\hline Halimeda tuna & $P_{I}$ & + & + & + & + & + \\
\hline Codium bursa & $P_{I}$ & Non & + & Non & + & + \\
\hline Cymodocea nodosa & $P_{I}$ & + & + & + & + & + \\
\hline \multicolumn{7}{|l|}{ Eponges } \\
\hline Chondrosia reniformis & $F$ & + & + & + & + & + \\
\hline Acanthella acuta & $F$ & + & + & + & + & + \\
\hline Petrosia sp. & $F$ & + & + & + & + & + \\
\hline \multicolumn{7}{|l|}{ Cnidaires } \\
\hline Anemonia sulcata & $C$ & + & + & - & + & + \\
\hline Actinia equina & $C$ & + & + & + & + & + \\
\hline Parazoanthus axinellae & $C$ & + & + & + & + & + \\
\hline Cerianthus sp. & C & Non & + & Non & - & + \\
\hline $\begin{array}{c}\text { Annélide } \\
\text { Sabella spallanzanii }\end{array}$ & $F$ & + & + & - & + & + \\
\hline \multicolumn{7}{|l|}{ Mollusques } \\
\hline Mytilus galloprovincialis & $F$ & Non & + & Non & + & + \\
\hline Patella coerulea & $H$ & + & + & - & + & + \\
\hline Littorina neritoïdes & $H$ & + & + & + & + & + \\
\hline \multicolumn{7}{|l|}{ Echinodermes } \\
\hline Paracentrotus lividus & $H$ & Non & + & Non & + & + \\
\hline Sphaerechinus granularis & $H$ & Non & + & Non & + & + \\
\hline Echinaster sepositus & $C$ & Non & + & Non & + & + \\
\hline Holothuria sp. & $D$ & + & + & + & + & + \\
\hline $\begin{array}{c}\text { Ascidie } \\
\text { Halocynthia papillosa }\end{array}$ & $F$ & + & + & + & + & + \\
\hline
\end{tabular}


La connaissance de la Mer :

un vecteur du développement durable en Méditerranée

La littérature sur le sujet est peu abondante et les seules études disponibles concernent l'herbier de posidonie. Des essais réalisés en laboratoire et in situ (SANCHEZ-LIZASO et al., 2008; GACIA et al., 20007) semblent montrer la sensibilité importante des posidonies (ainsi qu'une plus faible abondance de la macrofaune associée) à une élévation de la salinité avec un seuil théorique voisin de 39. Nous n’avons pas testé l'effet du sel sur la posidonie mais sur une autre phanérogame marine, la cymodocée. Celle-ci semble supporter l'augmentation de salinité (jusqu’à 49-50) au moins pour la durée de l'expérimentation.

D’une façon plus générale, la plupart des organismes peuvent s'adapter à des variations mineures des conditions optimales de salinité et peuvent tolérer temporairement des situations extrêmes. Cela ne présage toutefois pas des changements ou altérations qui pourraient intervenir dans le cas d'une exposition permanente.Une salinité de 48 à 49 semble également pouvoir affecter le recrutement naturel (moules, patelles ou acétabulaires).

Au niveau de la méiofaune du sédiment des différences de comportement vis à vis de l'augmentation de salinité ont pu être mises en évidence. Les espèces les plus sensibles sont les annélides et les copépodes. A l'inverse, les nématodes apparaissent résistants et deviennent majoritaires en fin d'expérience. Ces résultats sont à rapprocher de ceux de DEL PILAR RUSO et al. (2007) qui observent une prépondérance des nématodes (98\%) à proximité d'un rejet de saumures pour des valeurs de salinité supérieures à 39.

Ces derniers résultats sont en définitive les plus représentatifs d'une élévation de la salinité, cette élévation se traduisant par une prédominance de nématodes au détriment d'autres groupes comme les annélides ou les copépodes. En effet au niveau du macrobenthos, seuls les cnidaires de type anémones sont les seuls à pouvoir être considérés comme indicateurs du fait de leur abondance en bordure littorale.

\section{Références bibliographiques}

DEL PILAR RUSO Y., DE LA OSSA CARRETERO J.A., GIMÉNEZ CASALDUERO F., SÁNCHEZ LIZASO J.L. (2007). Spatial and temporal changes in infaunal communities inhabiting soft-bottoms affected by brine discharge. Marine Environmental Research, Volume 64(4), pp 492-503. doi:10.1016/j.marenvres.2007.04.003 GACIA E., INVERS O., MANZANERA M., BALLESTEROS E., ROMERO J., (2007). Impact of the brine from a desalination plant on a shallow seagrass (Posidonia oceanica) meadow. Estuarine, Coastal and Shelf Science, Volume 72(4), pp 579-590. doi:10.1016/j.ecss.2006.11.021

SANCHEZ-LIZASO J.L., ROMERO J., RUIZ J., GACIA E., BUCETA J.L., INVERS O., FERNANDEZ TORQUEMADA Y., MAS J., RUIZ-MATEO A., MANZANERA M. (2007). Salinity tolerance of the Mediterranean seagrass Posidonia oceanica: recommendations to minimize the impact of brine discharge from desalination plants. Desalination 221 pp 602-607. doi:10.1016/j.desal.2007.01.119 